\title{
Performance evaluation of acoustic
}

\section{underwater data broadcasting exploiting the bandwidth-distance relationship}

\author{
P. Nicopolitidis ${ }^{\mathrm{a}, *}$, K. Christidis ${ }^{\mathrm{a}}$, G.I. Papadimitriou ${ }^{\mathrm{a}}$, P.G. Sarigiannidis ${ }^{\mathrm{b}}$ and \\ A.S. Pomportsis ${ }^{\mathrm{a}}$ \\ ${ }^{a}$ Department of Informatics, Aristotle University of Thessaloniki, Box 888, 54124, Thessaloniki, Greece \\ ${ }^{\mathrm{b}}$ Department of Engineering Informatics and Telecommunications, University of Western Macedonia, \\ 50100, Kozani, Greece
}

\begin{abstract}
Despite being a fundamental networking primitive, data broadcasting has so far received little attention in the context of underwater networks. This paper proposes an adaptive push system for data broadcasting in underwater acoustic wireless networks with locality of client demands. The proposed system exploits the characteristic relationship between the bandwidth of an underwater acoustic link and the transmitter-receiver distance in order to improve performance in environments with locality of client demands. Simulation results show superior performance of the proposed approach in the underwater environment compared to existing systems.
\end{abstract}

Keywords: Underwater networks, acoustic communications, data broadcasting, locality of demand

\section{Introduction}

The underwater environment poses a number of unique challenges for implementing communication. Due to the fact that radio waves and optical beams cannot easily be applied in underwater, the transmission method of choice for the physical layer of underwater environment is the use of acoustic waves. Underwater acoustic communications entails a number of interesting characteristics, with the most important ones being the Bit Error Rate, the propagation delay and the typical Bandwidth-distance relationship exhibited by such links. Specifically, a) the Bit Error Rate of underwater acoustic links is higher than that of terrestrial radio-based ones, b) the low speed of sound in the water $(1.5 \mathrm{Km} / \mathrm{sec})$ gives a propagation delay that is five orders of magnitude larger than that of terrestrial radio-based links of the same length and c) there exists a relationship between the maximum coverage area of an underwater acoustic transmitter and both the centre frequency of the channel available to the transmitter and the channel's bandwidth. As the transmission distance increases, the operating frequency band must be shifted toward lower frequencies and its available bandwidth reduces. Typical values for this bandwidth range from several tens of $\mathrm{KHz}$ for areas of a few kilometres to several $\mathrm{Hz}$ for areas that span tens of kilometres.

\footnotetext{
${ }^{*}$ Corresponding author. Tel.: +30 2310998538; Fax: +30 2310998417; E-mail: petros@csd.auth.gr.
} 
Despite being a popular networking primitive, data broadcasting $[16,18,19,25-30]$ has so far received little attention in the context of underwater networks with the only relevant work presented in [20, 21,23]. [20] proposes three reliable broadcast protocols based on the capabilities of Forward Error Correction (FEC). The Bandwidth-distance relationship of the underwater acoustic environments allows clients to forward the server's broadcast to clients further away. More concretely, this is accomplished by employing specific frequency bands where the signals are not expected to travel long distances. This way a considerable reduction of the number of transmissions required to complete the broadcast is achieved, which in turn reduces both the overall energy consumption and the total time it takes to complete the broadcast. A quite different approach is presented in [21] which makes use of Fountain codes to enhance the efficiency of the data dissemination process in the face of poor channel conditions. The proposed broadcasting scheme can provide a trade-off between different performance metrics such as delay, advancement per hop and transmission power.

Data broadcasting in the underwater acoustic environment to a number of clients with overlapping demands for data items, has been addressed in [23]. Apart from achieving adaptation of its broadcast schedule according to the a-priori unknown needs of the clients, the proposed system also efficiently combats the problem of high latency of the underwater acoustic wireless environment. Simulation results in [23] show superior performance of the proposed system in the underwater environment compared to adaptations of existing terrestrial push systems.

This paper proposes an adaptive push-based data broadcasting system that extends the above-mentioned method in environments where there is a need for transmission of data items to multiple underwater clients that exhibit locality of demand [27-29]. The latter means that clients are grouped into groups, each one located at a different place with members of each group having similar demands, different from those of clients at other groups. Examples of such scenarios include situations where underwater groups of clients (e.g. submarines or divers or even sensors) are positioned at different locations inside a server's coverage area. These groups demand specific information from a Base Station, which is essentially the Broadcast Server. The terrain map for the group's location for example, is information, which differs according to the location of the group, and even if it is crucial and popular for one group it remains completely useless for another.

The topology of the proposed system comprises groups of clients that exhibit locality of demand for items and a Base Station with acoustic transmission capabilities, all with full-duplex acoustic modems as already proposed in other acoustic underwater protocols [11,17]. The Base Station from its side undertakes the communication with the terrestrial data repository either via a cable or a radio-wireless link. A Learning Automaton [14] is being used by the Base Station in order to estimate the overall transmission probability of each data item, by continuously adapting to the demand pattern of the client population via simple feedback received from the clients. Compared to its predecessor [23], the proposed system increases performance due to exploitation of locality of demand via the bandwidthdistance relationship.

Underwater acoustic wireless networks can support a large variety of applications. The proposed system can provide support for several situations. The Base Station can disseminate data items that contain graphical and contextual information (e.g. map, objects and temperature currents respectively). Some examples are maritime communications, environmental and equipment monitoring (e.g. tsunami and earthquake detection), manned missions such as monitoring of stations or divers who are equipped with underwater GPS, (or another positioning method [3-5,15] and an acoustic navigation system aiming at the mapping and positioning of the diver. Having knowledge of its current position, the equipment (e.g. [13]) of each diver will expect to receive and thus acknowledge only data items that contain useful information regarding its present position. 
The context of this paper is organized as follows. Section 2 describes the proposed underwater data broadcasting system for environments with locality of client demands. Simulation results that reveal the superiority of the proposed system are presented in Section 3. Finally, Section 4 concludes this paper.

\section{The proposed push system}

\subsection{The broadcasting algorithm-basics}

The broadcasting algorithm operates as follows: The Broadcast Server is equipped with an S-model Linear Reward-Inaction Learning Automaton (LA) that contains the server's estimate $p_{i}$ of the actual demand probability $d_{i}$ of the client population for each data item $i$ among the set of the items the server broadcasts. Learning Automata $[7,8,14]$ are mechanisms that can be applied to learn the characteristics of a system's environment. In the area of data networking, Learning Automata have been applied to several problems, including the design of self-adaptive MAC protocols for wired and wireless platforms (e.g. $[9,10,24])$ and routing (e.g. $[1,2])$.

Clearly $\sum_{i=1}^{N} p_{i}=\sum_{i=1}^{N} d_{i}=1$, where $N$ is the number of items in the server's database. At each cycle the server will transmit the item $i$ that maximizes the cost function $G(i)=(T-R(i))^{2} \frac{p_{i}}{l_{i}}\left(\frac{1+E\left(l_{i}\right)}{1-E\left(l_{i}\right)}\right)[16]$. In the above Equation $T$ is the current time, $R(i)$ the time when item $i$ was last broadcast, $l_{i}$ is the length of item $i$ and $E\left(l_{i}\right)$ is the probability that an item of length $l_{i}$ is erroneously received. For items that haven't been previously broadcast $R(i)$ is set to -1 . If the maximum value of $G(i)$ is shared by more than one item, the algorithm selects one of them randomly.

The server will probe the data items in order to receive feedback from them so as to estimate the demand probability for each data item. For the feedback transmission, CDMA, which has also been used in the underwater acoustic environment [6,12], is chosen. On the other hand, data item are broadcast via narrowband modulation over the entire available bandwidth for data broadcasting. The number of mobile clients that can be supported in the system is given by the capacity of CDMA, which is given by $\frac{W_{F B} / U_{F B}}{E_{b} / N_{o}}+1$. $W_{F B}$ represents the transmission bandwidth for the feedback, $U_{F B}$ represents the feedback transmission bit rate and $E_{b} / N_{o}$ is the bit energy-to-noise power spectral density.

To combat the problem of increased latency of the underwater links, which are underutilized by application of terrestrial stop-and-wait push systems [25], where link latency is insignificant, [23] proposes that a continuous data broadcasting approach is employed. To this end, the length of each client feedback will comprise a $1+\left\lceil\log _{2} N\right\rceil$ number of bits so as to code the id of the item is acknowledges. To collect feedback from the clients the server uses an in-band channel over the transmitted data items. This channel is realized by piggybacking two binary numbers, $\mathrm{A}$ and $\mathrm{B}$ on the of each item $i, 1 \leqslant i \leqslant N$. A is a one-bit number, while B comprises a $\left\lceil\log _{2} N\right\rceil$ number of bits. Two cases can be distinguished: a) the server does not request feedback after the broadcast of item $i$, it notifies the clients for this by setting $\mathrm{A}=0$ and $\mathrm{b}$ ) the server wants to collect feedback by the clients that are currently waiting to receive item $j$, it sets on the header of the broadcast item $i, \mathrm{~A}=1$ and $\mathrm{B}=\operatorname{decimal} j$ in order to probe item $j$. In order to select which item to probe, the cost function $\mathrm{G}$ is separately applied to produce the sequence of probed items, assuming that time elapses only when transmissions of items with probing requests are made. This means that the server needs to store a separate vector $R^{\prime}$ for keeping the time when each item was last probed by the server. 
After the transmission of an item that requests feedback response, the server will continuously broadcast items for a time interval equal to the duration of the feedback transmission. For this interval, clients who successfully received data items, will read the binary flag $\mathrm{A}=0$ at the item's header and will not respond with a feedback.

When feedback arrives at the server, the latter will read the item id on the incoming feedback and uses it to update the estimation of the corresponding items. The Learning Automaton, which maintains the probability distribution vector $p$, estimates the demand probability $d_{i}$ of each information item $i$. Assuming item $j$ is the server's $k^{\text {th }}$ transmission, the following Linear Reward-Inaction $\left(L_{R-I}\right)$ probability updating scheme [14] is used whenever a feedback for item $j$ is received at the server:

$$
\begin{aligned}
& p_{m}(k+1)=p_{m}(k)-L\left(\frac{p_{m}(k)-a}{C l N u m}\right), \forall j \neq m \\
& p_{j}(k+1)=p_{j}(k)+\left(\frac{L}{C l N u m}\right) \sum_{m \neq j}\left(p_{m}(k)-a\right)
\end{aligned}
$$

where $L, a$ take values in $(0,1)$ and $p(k)$ takes values in $(a, 1)$. Thus, the probabilities of the items do not change when the probing of an item $j$ does not satisfy any waiting client, whereas following a broadcast that satisfies clients, the probability of item $j$ is increased. To detect the actual number of clients, ClNum, the server can broadcast a control item that forces every client to respond with a feedback and then waits for feedbacks for a time period equal to the time needed by a client a the maximum coverage distance from the server to receive the control packet and send its feedback to the server. $L$ defines the speed of convergence. The lower the value of $L$, the more accurate the estimation made by the automaton, a fact that comes at expense over convergence speed. Parameter $a$ prevents the probabilities of less popular items from taking values close to zero increasing the adaptability of the automaton. Using this scheme, convergence of the item probabilities estimated by the Automaton to the actual overall demand pattern of the client population is achieved [23,25-29].

\subsection{Exploiting the bandwidth-distance relationship}

As already mentioned above, there exists a fundamental relationship between the distance of the transmitter and the receiver and both the centre frequency of the channel available to the transmitter and the channel's bandwidth. This relationship between bandwidth and distance of an underwater acoustic channel has been extensively examined in [21] and is summarized in Fig. 1 for transmitter-receiver distances that range between 0 and $5 \mathrm{Km}$. The curve represents the channel's server frequency in $\mathrm{KHz}$ while the vertical bars represent the available bandwidth for the respective transmitter-receiver distances.

The above-mentioned relationship can be exploited in order to provide a performance increase to the system. In order to quantify this, we present the following example. Consider a system which does not employ the bandwidth-distance relationship, equipped with a server that acoustically transmits items to underwater clients located inside the server's coverage area of a $5 \mathrm{Km}$ radius. According to Fig. 1, in order to provide coverage to all clients inside the $5 \mathrm{Km}$ range, the server will use a channel with bandwidth about $10 \mathrm{KHz}$, which we split into $8 \mathrm{KHz}$ for item transmission and $2 \mathrm{KHz}$ for feedback transmission in order to support a certain number of clients according to the capacity equation of CDMA. So, the transmission to each client is made via the same bandwidth and as a result with the same data rate.

To further increase performance, we exploit the bandwidth-distance relationship of underwater acoustic links in order to decrease the item transmission time by transmitting at higher rates to clients located 


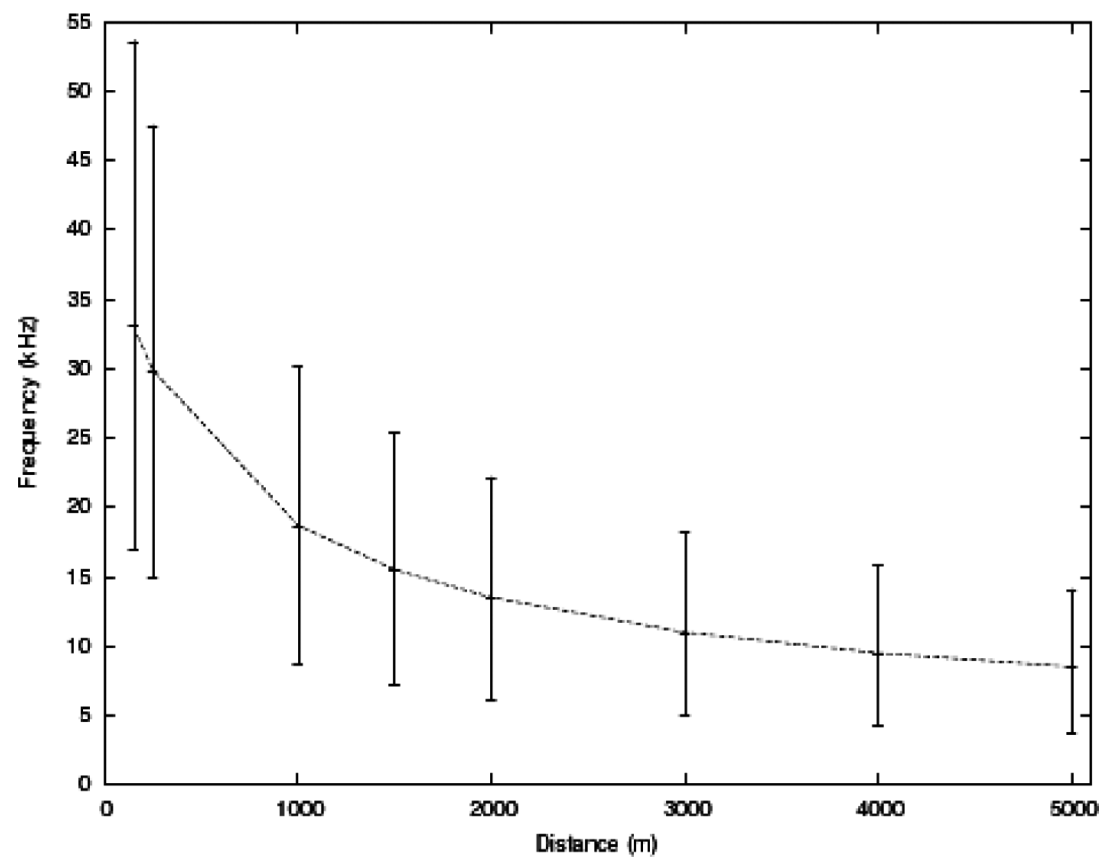

Fig. 1. The effect of distance on the channels center frequency and its available bandwidth (reported in [21]).

closer to the Broadcast Server. Reverting to the previous example and taking into consideration the effect of distance on the available bandwidth (Fig. 1), the server transmission channel for a group of clients located at a distance of $1 \mathrm{Km}$ will be set at around $22 \mathrm{KHz}$. If we again use $2 \mathrm{KHz}$ to support the same number of clients over the feedback channel, the remaining $20 \mathrm{KHz}$ for data item transmissions provide a two-fold increase in the transmission rate. The closer a client is located to the server, the higher the data rate that will be used.

By using the data from Fig. 1 and application of interpolation for distances up to $5 \mathrm{Km}$ and extrapolation for longer distances, we can obtain the corresponding channel bandwidths for several transmitter-receiver distances. Since the server a) possesses knowledge of its position, b) it broadcasts data items that concern the environmental surroundings at certain distances and c) it knows which items contain information that concern specific positions at specific distances, it will be able to broadcast data items to different areas at different channels that are determined from the server's distance to that area. The underwater clients on the other hand can pinpoint their position via their on-board location mechanism [3-5,22] and since the position of the server is known, each client can compute its distance from the server and thus determine the channel (center frequency and bandwidth) over which it will receive data from the server.

\section{Performance evaluation}

In order to assess the performance increase of the proposed approach, we used an event-driven simulator coded in $\mathrm{C}++$ to compare it to the underwater data broadcasting system of [23] and to the stop-and-wait approach of [25] adapted to the underwater environment. We consider a broadcast server having a database of $N$ equally-sized items. The server is initially unaware of the demand for each item, so in the beginning of simulation process, every item has a probability estimate $p_{i}$ of $1 / N$. In the systems of [23, 
Table 1

Group positions uniformly distributed

\begin{tabular}{lccccc}
\hline & $\begin{array}{c}\text { Group 1 } \\
\text { distance }(\mathrm{Km})\end{array}$ & $\begin{array}{c}\text { Group 2 } \\
\text { distance }(\mathrm{Km})\end{array}$ & $\begin{array}{c}\text { Group 3 } \\
\text { distance }(\mathrm{Km})\end{array}$ & $\begin{array}{c}\text { Group 4 } \\
\text { distance }(\mathrm{Km})\end{array}$ & $\begin{array}{c}\text { Group 5 } \\
\text { distance }(\mathrm{Km})\end{array}$ \\
\hline MAX_DISTANCE 2 Km & 0.4 & 0.8 & 1.2 & 1.6 & 2.0 \\
MAX_DISTANCE 5 Km & 1 & 2 & 3 & 4 & 5 \\
\hline
\end{tabular}

25], a fixed bit-rate is used, so the server broadcasts all items with the same bit rate. The proposed system with exploitation of the bandwidth-distance relationship is a variable bit-rate system because the server determines the bit rate to use according to the server-client distance. As far as the number of feedback bits are concerned, one-bit feedback is used for the method of [25] while $1+\left\lceil\log _{2} N\right\rceil$ bits are needed for the feedback of the proposed approach and that of [23].

We consider ClNum underwater clients that due to the time volatile nature of item contents, have no cache memory - an assumption also made in other similar research both for terrestrial and underwater environments (e.g. [16,23,25-29]). Clients are grouped into $G$ groups each one of which is located at a different distance from the antenna. Any client belonging to group $g, 1 \leqslant g \leqslant G$ is interested in the same subset $S e c_{g}$ of the server's database. All items outside this subset have a zero demand probability at the clients of the group. Finally, all subsets are of the same size and $S e c_{i} \neq S e c_{j}, \forall i, j \in[1, \ldots, G], i \neq j$.

To simulate some disagreement in the client demand patterns, we introduce the parameters Dev and Noise. A coin toss weighted by Dev, is made for all clients. If the outcome of the toss states that the client will deviate from the initial demand pattern, then a new pattern for this client is created. This pattern is produced as follows: with probability Noise the demand probability of each item in the client's demand pattern database is swapped with another item that is selected in a uniform manner from the interval $[1 \ldots N]$.

The simulation is carried out until at least Numreq requests are satisfied at each client, meaning that overall, at least $N u m r e q \times C l N u m$ requests have been served. The following constant parameter values were used for the simulation: $N=500, G=5, N u m r e q=5000, L=0.15, a=10^{-4}$. Dev and Noise parameter when set to non-zero take values of 0.3 and 0.5 respectively. Item size is set to $10^{3}$ bits, $C l N u m=81$ and $E_{b} / N_{0}=5$. Finally, the speed of sound in the water is set to $1.5 \mathrm{Km} / \mathrm{sec}$.

The Database subsets accessed by the different client groups are chosen as follows: Each of the 5 groups demands the following sections of items respectively: $S e c_{1}=[1 \ldots 200], S_{e c}=[201 \ldots 350]$, $S_{e c}=[351 \ldots 400], S_{3} c_{4}=[401 \ldots 450], S e c_{5}=[451 \ldots 500]$. Each group g is placed at $x$ kilometers from the server, and we assume that all clients of this group have this same distance. Table 1 shows the distance of each of the 5 groups from the Broadcast Server. Assuming that each $S e_{i}$ subset comprises Num items, the demand probability $d(i)$ of an item in region $i$ in that subset is calculated according to the following Zipf distribution as $c\left(\frac{1}{i}\right)^{\theta}$, where $c=1 / \sum_{k}\left(\frac{1}{k}\right)^{\theta}, k \in[1 \ldots N u m]$. The Zipf distribution, which is used in other relative papers as well $[16,23,25-29]$ can efficiently model applications that are characterized by a certain amount of commonality in client demands. $\theta$ is a parameter named access skew coefficient. For $\theta=0$, the Zipf distribution reduces to a uniform distribution of demand for the items in a subset, while as the value of $\theta$ increases, increasingly skewed demand patterns are produced. Finally, we did not take into consideration reception errors as the goal is to present the relative performance of the compared systems.

For the system of [23] we used an available bandwidth for broadcasting and feedback channel implementation of $W_{D A T A}=8 \mathrm{KHz}$ and $W_{F B}=2 \mathrm{KHz}$ respectively. Consequently, the broadcasting rate and the feedback transmission rate were set to $U_{D A T A}=1900 \mathrm{bps}$ and $U_{F B}=5$ bps respectively. These values correspond to a distance of $10 \mathrm{Km}$. For server coverage areas of 5 and $2 \mathrm{Km}$ radius the 
Table 2

Parameters of simulation environments

\begin{tabular}{cccccc}
\hline & Clients in Group 1 & Clients in Group 2 & Clients in Group 3 & Clients in Group 4 & Clients in Group 5 \\
\hline N1 & $1 \ldots 16$ & $17 \ldots 32$ & $33 \ldots 48$ & $64 \ldots 49$ & $65 \ldots 81$ \\
N2 & $1 \ldots . .32$ & $33 \ldots 56$ & $57 \ldots 64$ & $65 \ldots 72$ & $73 \ldots 81$ \\
N3 & $1 \ldots 9$ & $10 \ldots 17$ & $18 \ldots . .25$ & $26 \ldots 49$ & $50 \ldots 81$ \\
\hline
\end{tabular}

MAX_DISTANCE 2Km Solid plots: Dev=0.0, Dashed plots: Dev $=0.3$

Client groups are in positions $0.4,0.8,1,1.6,2 \mathrm{Km}$

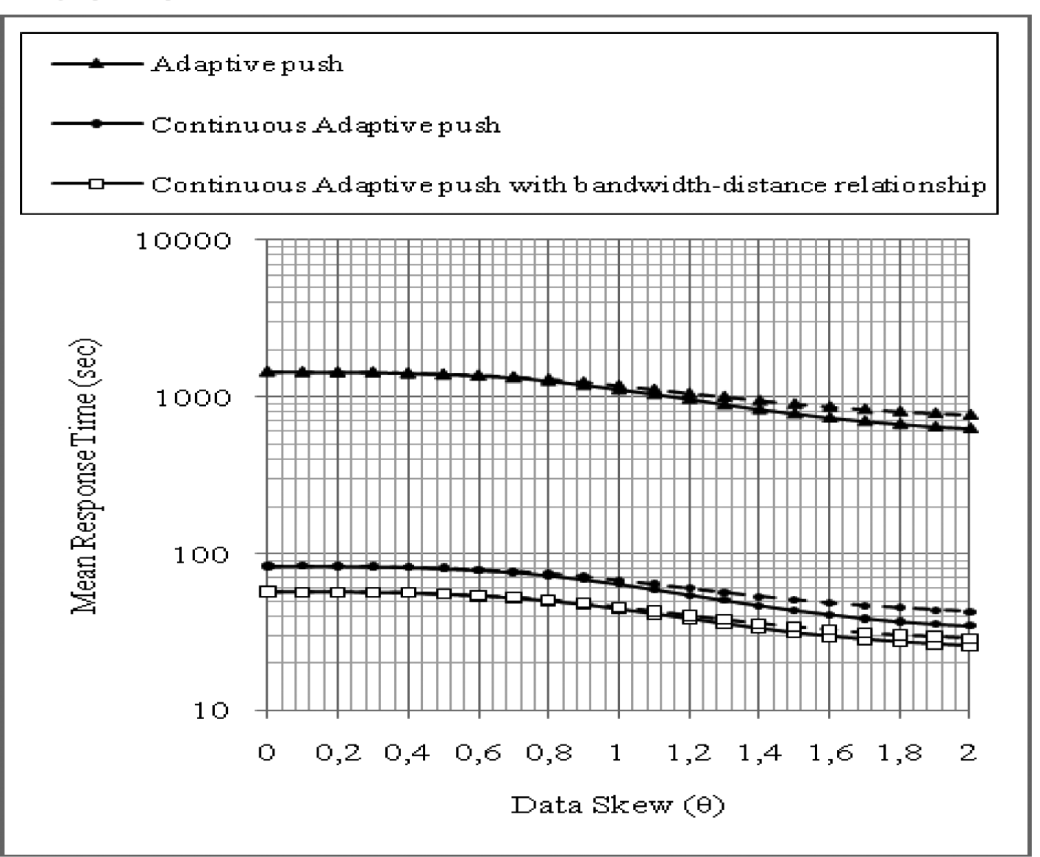

Fig. 2. Mean response time versus data access skew coefficient for the three systems in Network N2 for a server coverage area of $2 \mathrm{Km}$ radius.

server uses $W_{D A T A}$ of $9 \mathrm{KHz}$ and $10 \mathrm{KHz}$ respectively, with respective proportional increases in the item transmission speed. The above parameters via Eq. (2) allow for $C l N u m=81$. For the system of [25], which utilizes 1-bit feedback, for the same feedback duration, 81 clients can be supported with $W_{F B}=$ $200 \mathrm{~Hz}$ and $U_{F B}=0.5 \mathrm{bps}$. Thus for this system $W_{D A T A}=9.8 \mathrm{KHz}$ and consequently $U_{D A T A}=2352$ bps, with respective proportional increases in the item transmission speed for server coverage areas of 5 and $2 \mathrm{Km}$ radius.

Finally, for the proposed system with exploitation of the bandwidth-distance relationship, the values of $W_{D A T A}, U_{D A T A}, W_{F B}$ and $U_{F B}$ for broadcasting to a client located at the border of the coverage area are the same with the ones used for $[23,25]$. However for client groups closer to the Broadcast Server $W_{D A T A}$ and $U_{D A T A}$ result from Fig. 1 according to the group's distance from the server.

We compared the performances of the three approaches in the following three environments, whose characteristics are shown in Table 2. We can see that in Network N1 client groups equally sized, in Network N2 the bigger groups are positioned closer to the server and in Network N3 the smaller groups are positioned closer to the server.

Figures 2-9 present the simulation results for the proposed approach and those of $[23,25]$. In these Figures, the maximum coverage area of the server, (marked as MAX_DISTANCE in the Figures) is 


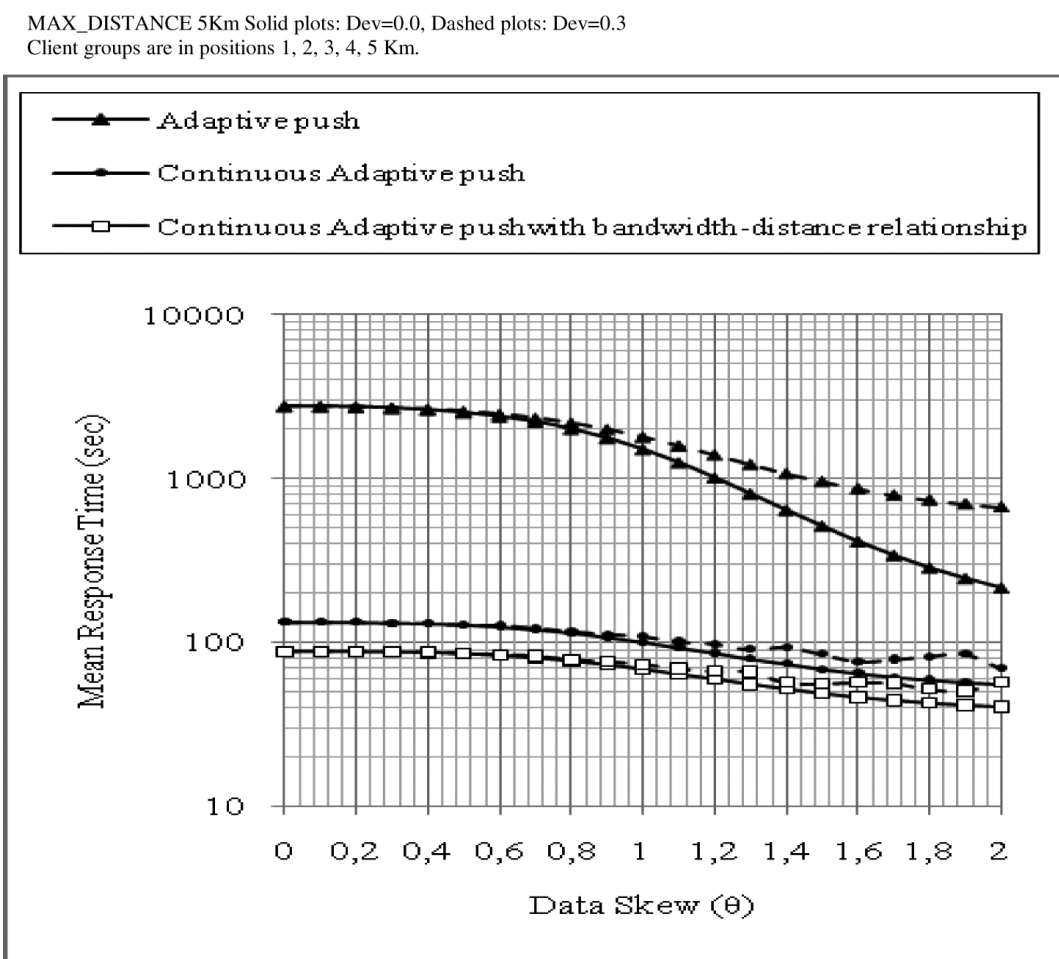

Fig. 3. Mean response time versus data access skew coefficient for the three systems in Network N2 for a server coverage area of $5 \mathrm{Km}$ radius.

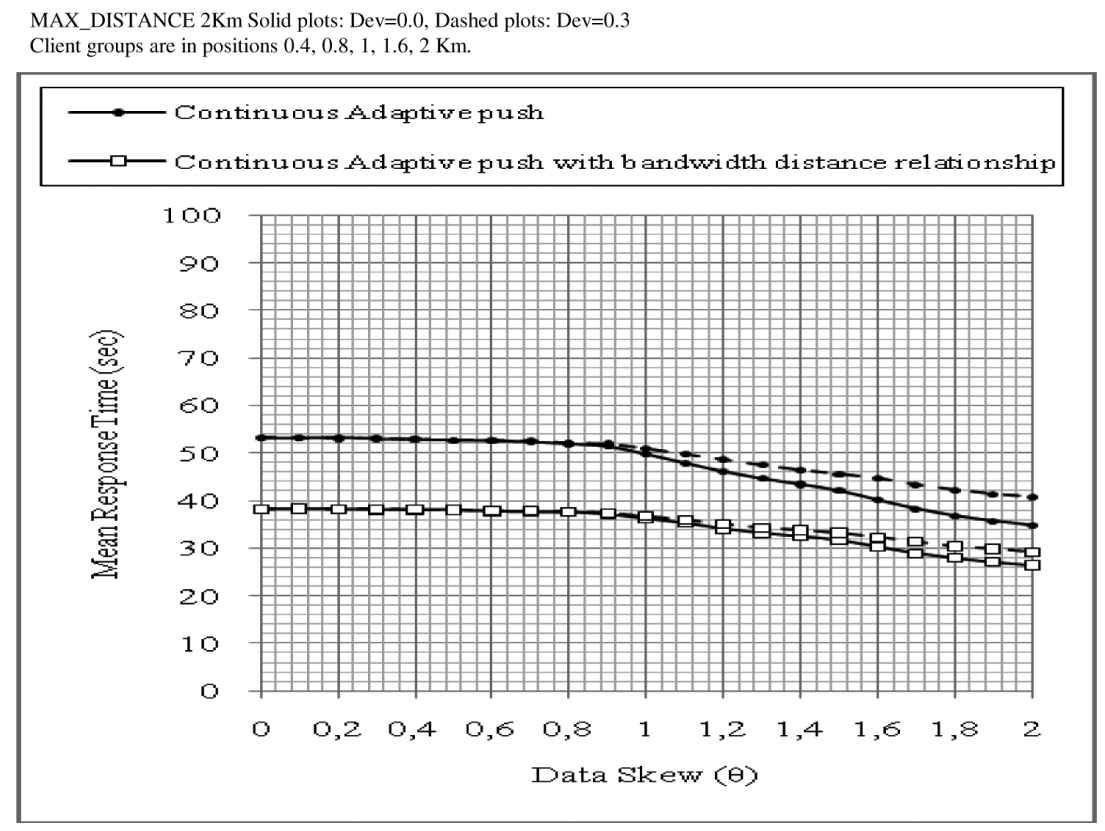

Fig. 4. Mean response time versus data access skew coefficient for the proposed and the continuous adaptive push system of [3] in Network N1 for a server coverage area of $2 \mathrm{Km}$ radius. 


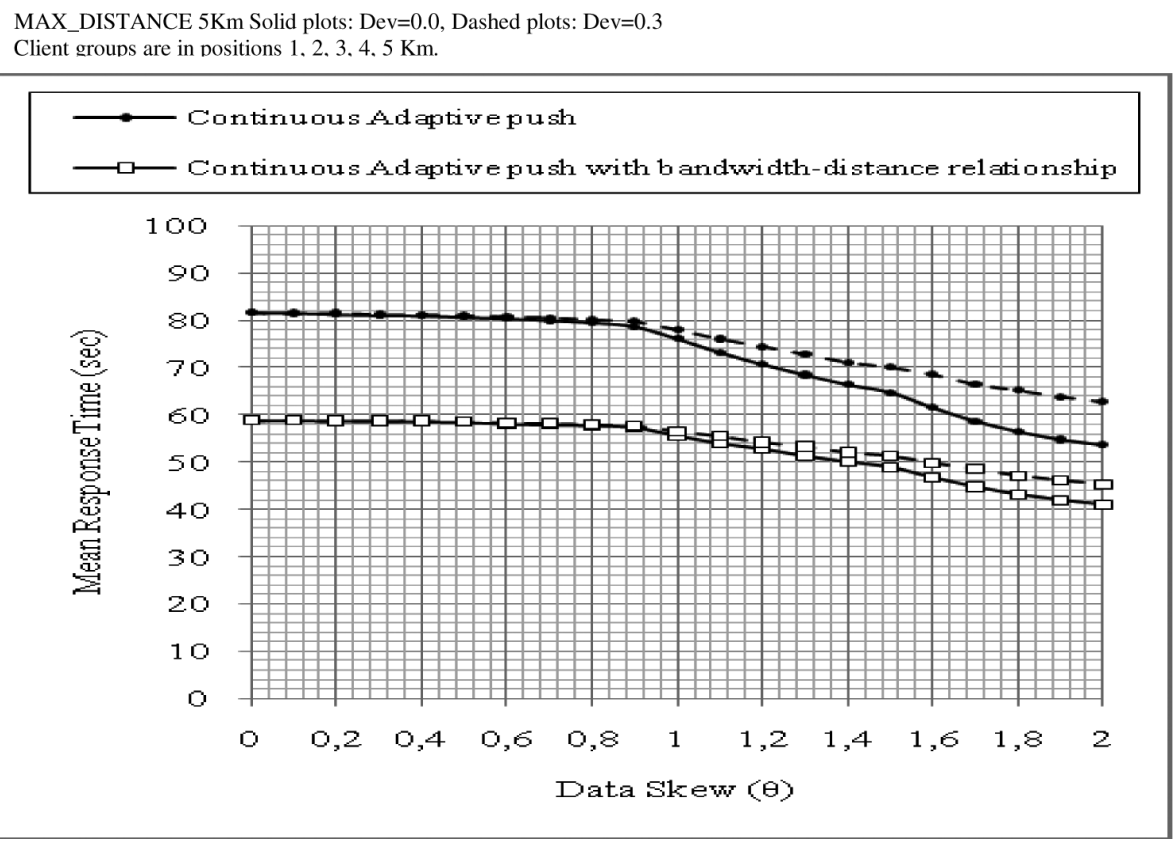

Fig. 5. Mean response time versus data access skew coefficient for the proposed and the continuous adaptive push system of [3] in Network $\mathrm{N} 1$ for a server coverage area of $5 \mathrm{Km}$ radius.

MAX_DISTANCE $2 \mathrm{Km}$ Solid plots: $\mathrm{Dev}=0.0$, Dashed plots: $\mathrm{Dev}=0.3$ Client groups are in positions $0.4,0.8,1,1.6,2 \mathrm{Km}$.

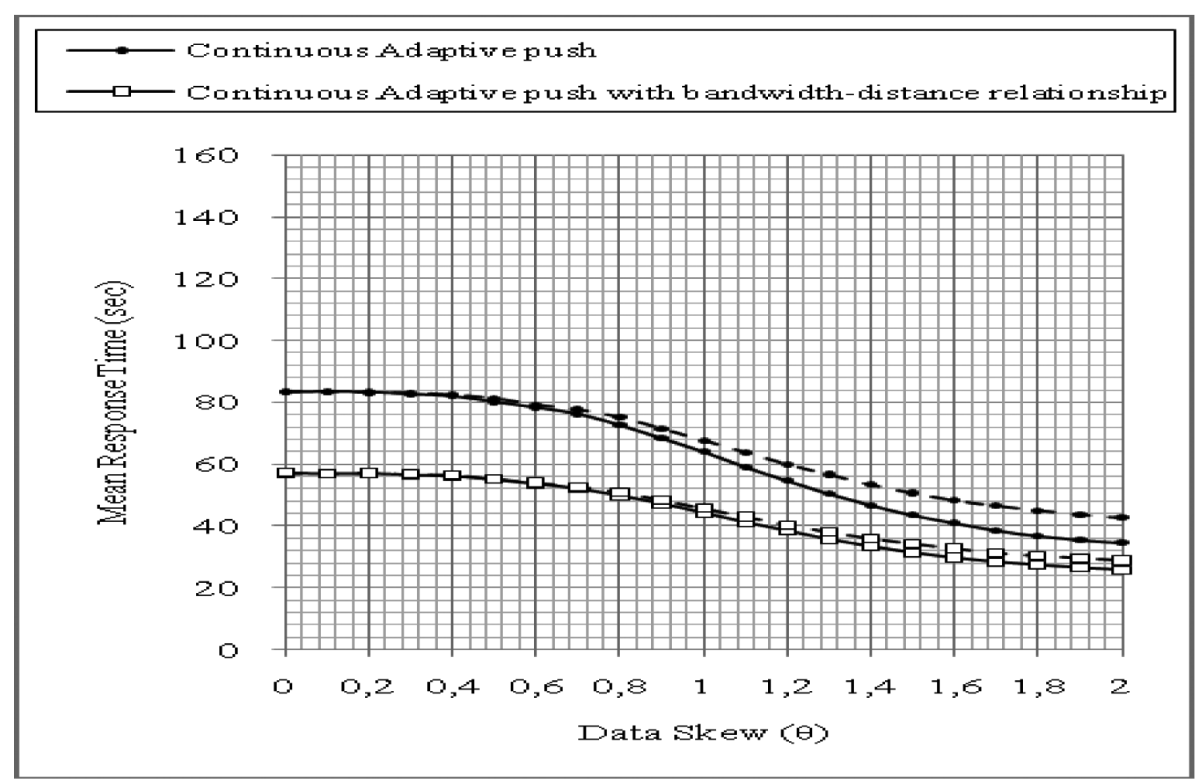

Fig. 6. Mean response time versus data access skew coefficient for the proposed and the continuous adaptive push system of [3] in Network $\mathrm{N} 2$ for a server coverage area of $2 \mathrm{Km}$ radius. 
MAX_DISTANCE $5 \mathrm{Km}$ Solid plots: Dev=0.0, Dashed plots: Dev $=0.3$

Client groups are in positions $1,2,3,4,5 \mathrm{Km}$.

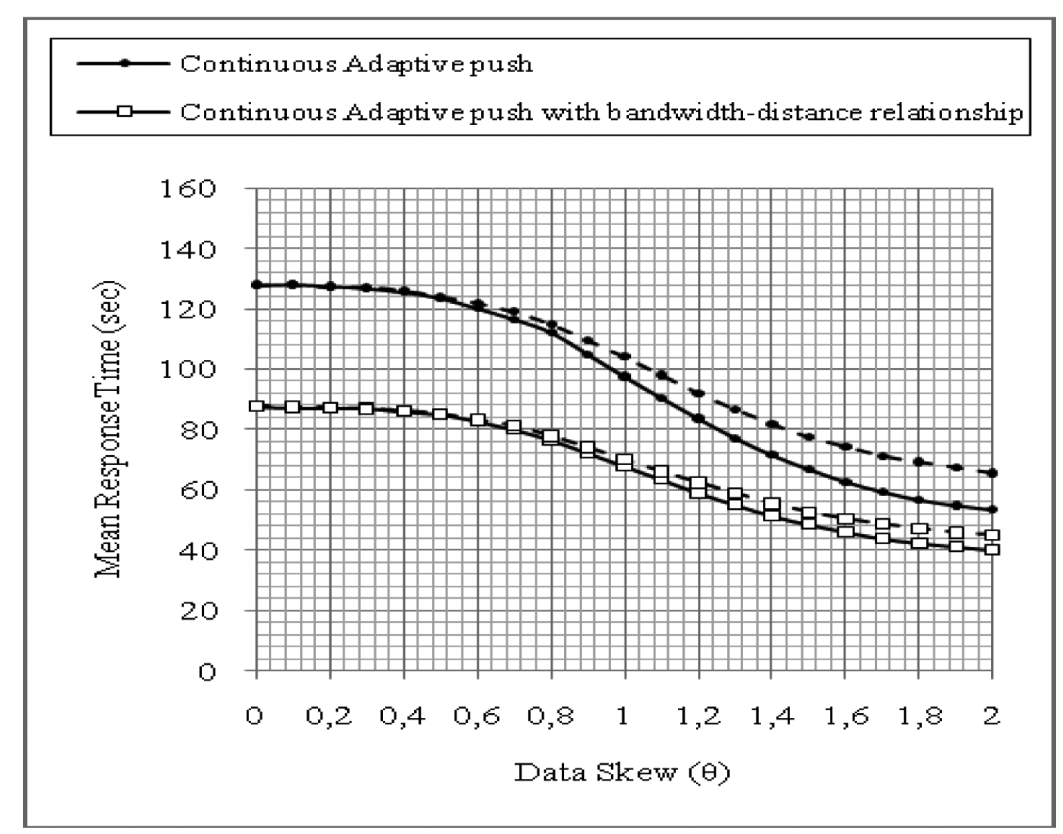

Fig. 7. Mean response time versus data access skew coefficient for the proposed and the continuous adaptive push system of [3] in Network $\mathrm{N} 2$ for a server coverage area of $5 \mathrm{Km}$ radius.

$$
\begin{aligned}
& \text { MAX_DISTANCE } 5 \mathrm{Km} \text { Solid plots: } \mathrm{Dev}=0.0 \text {, Dashed plots: } \mathrm{Dev}=0.3 \\
& \text { Client groups are in positions } 1,2,3,4,5 \mathrm{Km} \text {. }
\end{aligned}
$$

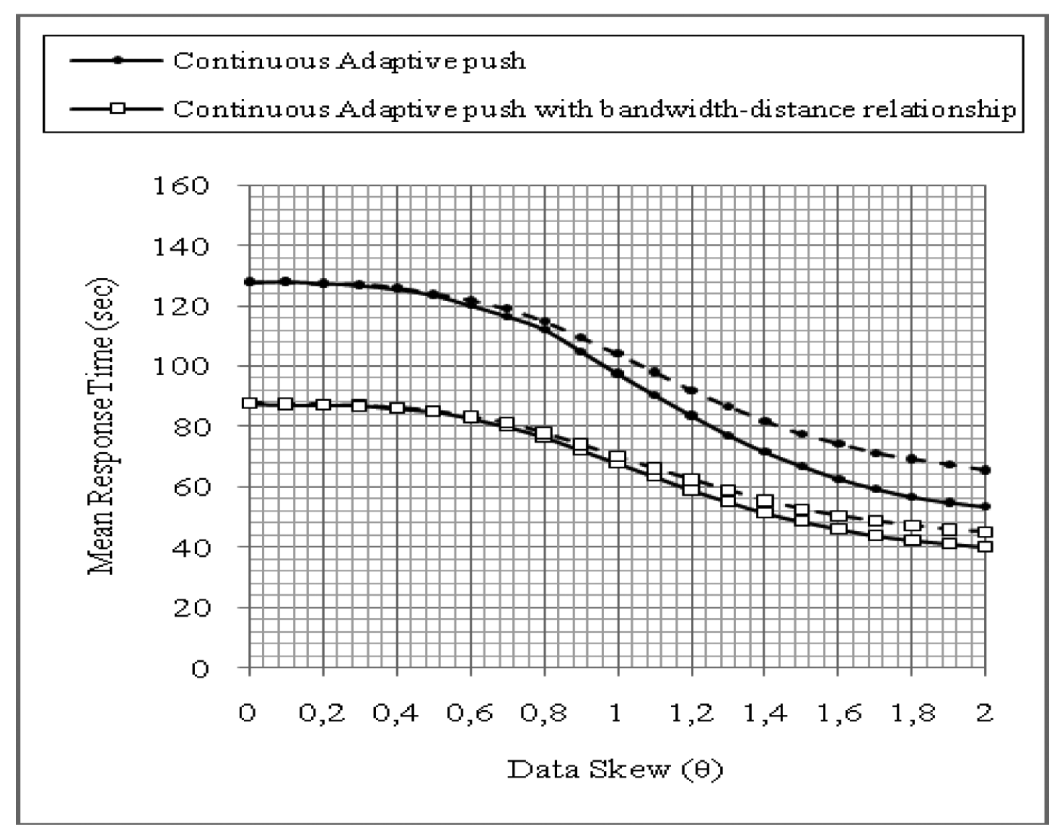

Fig. 8. Mean response time versus data access skew coefficient for the proposed and the continuous adaptive push system of [3] in Network N3 for a server coverage area of $2 \mathrm{Km}$ radius. 


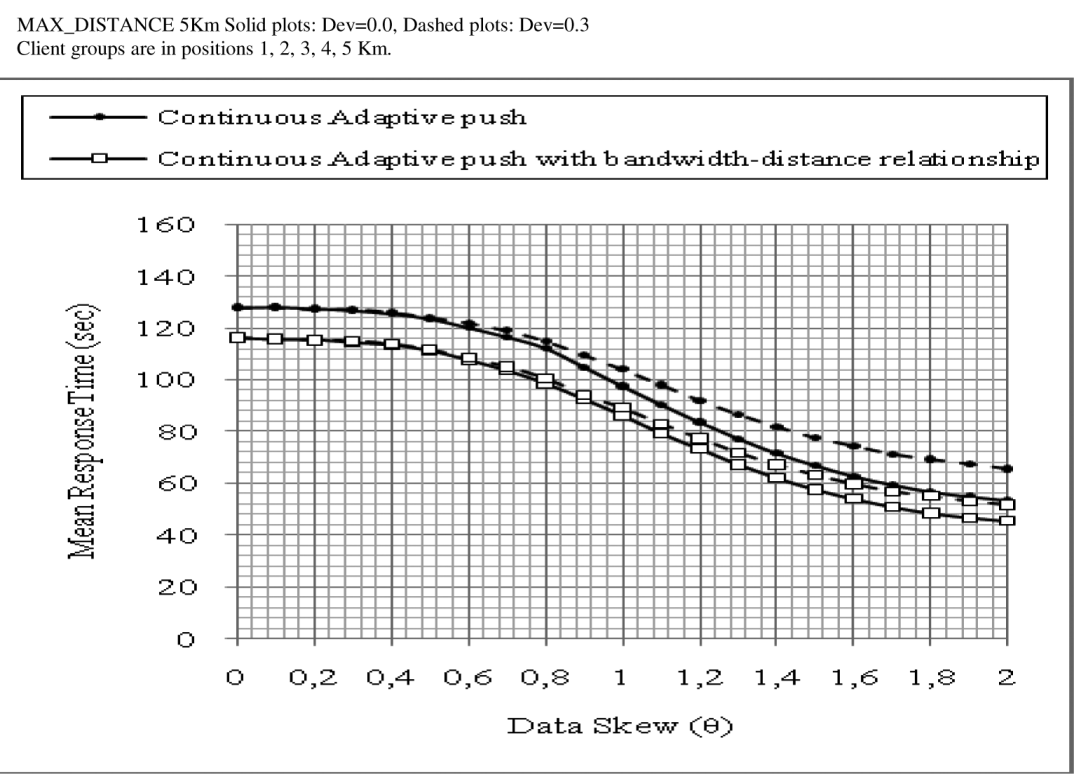

Fig. 9. Mean response time versus data access skew coefficient for the proposed and the continuous adaptive push system of [3] in Network N3 for a server coverage area of $5 \mathrm{Km}$ radius.

2 or $5 \mathrm{Km}$. Specifically, Figs 2, 3 compare the performances of the proposed approach to that of [23, $25]$ in Network N2 with a logarithmic (base 10) scale used for the Y-axis. The pairs of Figs 4, 5, 6, 7 and 8, 9 compare the performance of the proposed approach to that of [23] for Networks N1, N2 and N3 respectively. All these Figures plot the performance of the compared systems versus the data access skew coefficient $\theta$. The used metric is the clients mean response time, a performance metric widely used in data broadcasting (e.g. [16,23,25-29]). Client mean response time is the mean time a client waits in order to receive a requested item. In all Figures, the plots termed "Adaptive push", "Continuous adaptive push", "Continuous Adaptive push with bandwidth-distance relationship" correspond to the stop-and-wait approach of [25], to the underwater push system of [23] and the proposed approach with exploitation of the bandwidth-distance relationship respectively. In all Figures, dashed plots correspond to the performance of the systems that make use of disagreement in the client demands with $D e v=0.3$ and Noise $=0.5$, whereas the solid ones correspond to the performances of the systems with $D e v=0$. The main conclusions that can be drawn from the Figures are the following:

- The performance of all schemes improves for increasing values of the data skew parameter. This is expected behavior $[16,23,25-29]$ as the Learning-Automaton adaptation mechanism manages to learn the actual demand probabilities of the various information items and use these values on the selection of the item to broadcast. Moreover the performances of all approaches decrease for $D e v=$ 0.3 due to the less commonality in client demands [23,25-28].

- The approach of [25] has performance that is worse by orders of magnitude compared to that of the proposed one. This is because the adaptive push system of [25] waits after each item transmission for the sum of twice the maximum propagation delay plus the item transmission time plus the feedback duration in order to receive a feedback, which obviously explains the huge mean response time rates. Contrarily, the two other methods allow a continuous transmission of data items for feedback collection, which in turn hides the increased transmission latency that burdens the system for the 
first item transmission. The same result appeared in simulations for environments N1 and N3 and is the reason that the performance of [25] is omitted from results in Figs 4-9.

- The proposed system that exploits the bandwidth-distance relationship results in a significant performance increase over that of [23]. These gains are larger in environments, where the bigger groups are located closer to the Broadcast Server. However, even in environments like N3, where the biggest groups are furthest away for the Server, the proposed system achieves a significant performance increase via the exploitation of the bandwidth-distance relationship which yields higher data rates for clients located closer to the Server.

\section{Conclusion}

As the popularity of underwater wireless networks increases, data broadcasting has emerged as an efficient way of disseminating information to underwater clients, with a high degree of commonality in their demand patterns. In many cases, clients are grouped into several groups, each one in a different position, with the members of each group having similar demands. This paper proposes an adaptive push system for data broadcasting in underwater acoustic wireless networks with locality of client demands. It exploits the characteristic relationship between bandwidth of an underwater acoustic link and transmitter-receiver distance in order to provide increased performance. Simulation results show superior performance of the proposed approach in the underwater environment compared existing push systems.

\section{References}

[1] A.A. Economides, Learning Automata Routeing in Connection-oriented Networks, International Journal of Communication Systems 8(4) (July-August 1995), 225-237.

[2] A.A. Economides, P.A. Ioannou and J.A. Silvester, Decentralized Adaptive Routing for Virtual Circuit Networks Using Stochastic Learning Automata, in: Proceedings of IEEE INFOCOM 1988, New Orleans, USA, March 27-31 1988, pp. 613-622.

[3] A. Mahajian and M. Walworth, 3-D position sensing using the differences in the Timeof-Flights from a wave source to various receivers, IEEE Transactions on Robotics and Automation 17 (February 2001), 91-94.

[4] D. Moore, J. Leonard, D. Rus and S. Teller, Robust distributed network localization with noisy range measurements, in: Proceedings of Sensys, November 3-5, 2004, Baltimore, U.S.A., pp. 50-61.

[5] D.Niculescu and B.Nathi, "Ad hoc positioning system (APS)", In Proceedings of IEEE GLOBECOM 2001, San Antonio, U.S.A, November 25-29, 2001, pp.2926-2931.

[6] D. Pompili, T. Melodia and I.F. Akyildiz, A CDMA-Based Medium Access Control for Underwater Acoustic Sensor Networks, IEEE Transactions on Wireless Communications 8(4) (April 2009), 1899-1909.

[7] G.I. Papadimitriou, A New Approach to the Design of Reinforcement Schemes for Learning Automata: Stochastic Estimator Learning Algorithms, IEEE Transactions on Knowledge and Data Engineering 6(4) (August 1994), 649-654.

[8] G.I. Papadimitriou, Hierarchical Discretized Pursuit Nonlinear Learning Automata with Rapid Convergence and High Accuracy, IEEE Transactions on Knowledge and Data Engineering 6(4) (August 1994), 654-659.

[9] G.I. Papadimitriou and A.S Pomportsis, Learning Automata-Based TDMA protocols for Broadcast Communication Systems with Bursty Traffic, IEEE Communication Letters 4(3) (March 2000), 107-109.

[10] G.I. Papadimitriou and A.S. Pomportsis, Self-Adaptive TDMA Protocols for WDM Star Networks: A LearningAutomata-Based Approach, IEEE Photonics Technology Letters 11(10) (October 1999), 1322-1324,

[11] H.-H. Ng, W.-S. Soh and M. Motani, MACA-U: A Media Access Protocol for Underwater Acoustic Networks, in: Proceedings of IEEE GLOBECOM, New Orleans, USA, December 2008.

[12] H.X. Tan and W.K.G. Seah, Distributed CDMA-based MAC Protocol for Underwater Sensor Networks, in: Proceedings of IEEE LCN 2007, 15-18 October, 2007 Dublin Ireland, pp. 26-36.

[13] http://wetpc.com.au/html/technology/wearable.htm.

[14] K.S. Narendra and M.A.L. Thathachar, Learning Automata: An Introduction, Prentice Hall, 1989. 
[15] Kenneth and D. Frampton, Acoustic self-localization in a distributed sensor network, IEEE Sensors Journal 6 (2006), $166-172$.

[16] N.H. Vaidya and S. Hameed, Scheduling Data Broadcast In Asymmetric Communication Environments, Wireless Networks 5(3), 171-182.

[17] N. Chirdchoo, W.-S. Soh and K.-C. Chua, Aloha-Based MAC Protocols with Collision Avoidance for Underwater Acoustic Networks, in: Proceedings of IEEE INFOCOM, Anchorage, USA, May 2007.

[18] S. Acharya, M. Franklin and S. Zdonik, Dissemination-based Data Delivery Using Broadcast Disks, IEEE Personal Communications 2(6) (December 1995), 50-60.

[19] S.S. Manvi, M.S. Kakkasageri and J. Pitt, Multiagent Based Information Dissemination in Vehicular Ad-hoc Networks, Mobile Information Systems 5(4) (2009), 363-389.

[20] P. Casari and A.F. Harris III, Energy-efficient Reliable Broadcast in Underwater Acoustic Networks, in Proceedings of ACM WUWNet, Montreal, Canada, September 2007.

[21] P. Casari, M. Rossi and M. Zorzi, Towards Optimal Broadcasting Policies for HARQ based on Fountain Codes in Underwater Networks, in Proceedings of IEEE/IFIP WONS, Garmisch-Partenkirchen, Germany, January 2008.

[22] P. Fülöp, S. Imre, S. Szabó and T. Szálka, Accurate Mobility Modeling and Location Prediction Based on Pattern Analysis of Handover Series in Mobile Networks, Mobile Information Systems 5(3) (2009), 255-289.

[23] P. Nicopolitidis, G.I. Papadimitriou and A.S. Pomportsis, Adaptive Data Broadcasting in Underwater Wireless Networks, IEEE Journal of Oceanic Engineering 35(3) (July 2010), 623-634.

[24] P. Nicopolitidis, G.I. Papadimitriou and A.S. Pomportsis, Learning-Automata-Based Polling Protocols for Wireless LANs, IEEE Transactions on Communications 51(3) (March 2003), 453-463.

[25] P. Nicopolitidis, G.I. Papadimitriou and A.S. Pomportsis, Using Learning Automata for Adaptive Push-Based Data Broadcasting in Asymmetric Wireless Environments, IEEE Transactions on Vehicular Technology 51(6) (November 2002), 1652-1660.

[26] P. Nicopolitidis, G.I. Papadimitriou and A.S. Pomportsis, Continuous-flow Wireless Data Broadcasting, IEEE Transactions on Broadcasting 55(2) (June 2009), 260-269.

[27] P. Nicopolitidis, G.I. Papadimitriou and A.S. Pomportsis, Exploiting Locality of Demand to Improve the Performance of Wireless Data Broadcasting, IEEE Transactions on Vehicular Technology 55(4) (July 2006), 1347-1361.

[28] P. Nicopolitidis, G.I. Papadimitriou and A.S. Pomportsis, Multiple Antenna Data Broadcasting for Environments with Locality of Demand, IEEE Transactions on Vehicular Technology 56(5) (September 2007), 2807-2816.

[29] V. Kakali, G.I. Papadimitriou, P. Nicopolitidis and A.S. Pomportsis, A New Class of Wireless Push Systems, IEEE Transactions on Vehicular Technology 58(8) (October 2009), 2529-4539.

[30] Z. Mammeri, F. Morvan, A. Hameurlain and N. Marsit, Location-dependent Query Processing Under Soft Real-time Constraints, Mobile Information Systems 5(3) (2009), 205-232. 
Konstantinos Christidis obtained the BS and Msc degrees in computer science from the Department of Informatics, Aristotle University of Thessaloniki, Greece, in 2007 and 2009 respectively. His research interests are in the area of wireless communications and mobile computing.

Petros Nicopolitidis received B.S. and Ph.D. degrees in computer science from the Department of Informatics, Aristotle University of Thessaloniki, Greece, in 1998 and 2002, respectively. From 2004 to 2009 he was a Lecturer at the same Department, were he currently serves as an Assistant Professor. He has published more than 70 papers in international refereed journals and conferences. He is co-author of the book Wireless Networks (Wiley, 2003). His research interests are in the areas of wireless networks and mobile communications. Since 2007 he serves as an associate editor for the International Journal of Communication Systems published by Wiley. He is a Senior Member of IEEE.

Georgios Papadimitriou received the Diploma and Ph.D. degrees in Computer Engineering from the University of Patras, Greece in 1989 and 1994 respectively. From 1989 to 1994 he was a Teaching Assistant at the Department of Computer Engineering of the University of Patras and a Research Scientist at the Computer Technology Institute, Patras, Greece. From 1994 to 1996 he was a Postdoctorate Research Associate at the Computer Technology Institute. From 1997 to 2001 and 2001 to 2006 , he was a Lecturer and Assistant Professor respectively at the Department of Informatics, Aristotle University of Thessaloniki, Greece. Since 2006 he is an Assiociate Professor at the same Department. His research interests include optical networks, wireless networks, high speed LANs and learning automata. Prof. Papadimitriou is Associate Editor of five scholarly journals, including the IEEE Transactions on Systems, Man and Cybernetics-Part C, the IEEE Transactions on Broadcasting, and the IEEE Communications Magazine. He is co-author of the books "Multiwavelength Optical LANs" (Wiley, 2003) and "Wireless Networks" (Wiley, 2003) and co-editor of the book "Applied System Simulation" (Kluwer, 2003). He is the author of more than 150 refereed journal and conference papers. He is a Senior Member of IEEE.

Panagiotis Sarigiannidis ( $\mathrm{S}^{\prime} 05-\mathrm{M}^{\prime} 07$ ) received the diploma and $\mathrm{Ph} . \mathrm{D}$. degrees in computer science from the Aristotle University of Thessaloniki, Greece, in 2001 and 2007, respectively. He is currently an adjunct Lecturer at the University of Western Macedonia, Greece. His research interests include wireless networks, mobile computing, optical networks and optical switching.

Andreas Pomportsis received the B.S. degree in physics and the M.S. degree in electronics and communications from the University of Thessaloniki, Greece, and the Diploma degree in electrical engineering from the Technical University of Thessaloniki, Greece. In 1987, he received the Ph.D. degree in computer science from the University of Thessaloniki. Currently, he is a Professor at the Department of Informatics, Aristotle University of Thessaloniki, Greece. He is coauthor of the books Wireless Networks (New York: Wiley, 2003) and Multiwavelength Optical LANs (New York: Wiley, 2003). His research interests include computer networks, learning automata, computer architecture, parallel and distributed computer systems, and multimedia. 

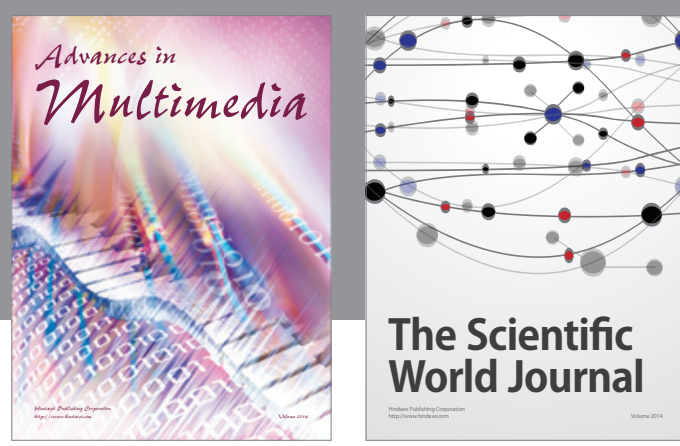

The Scientific World Journal
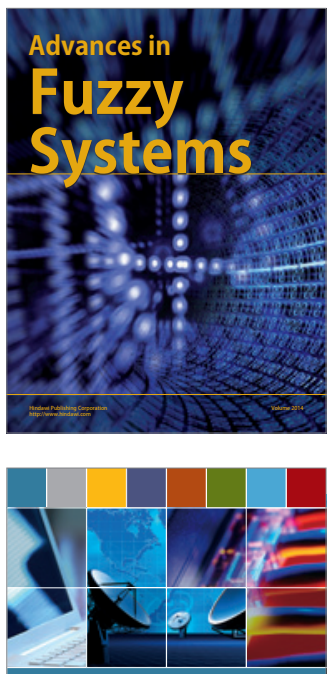

Computer Networks and Communications
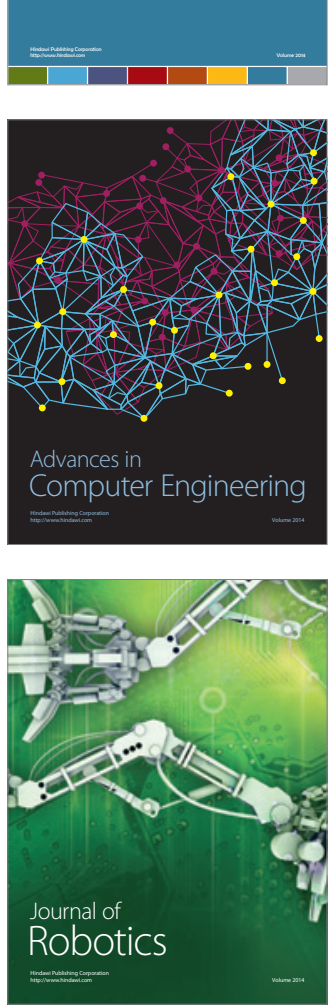
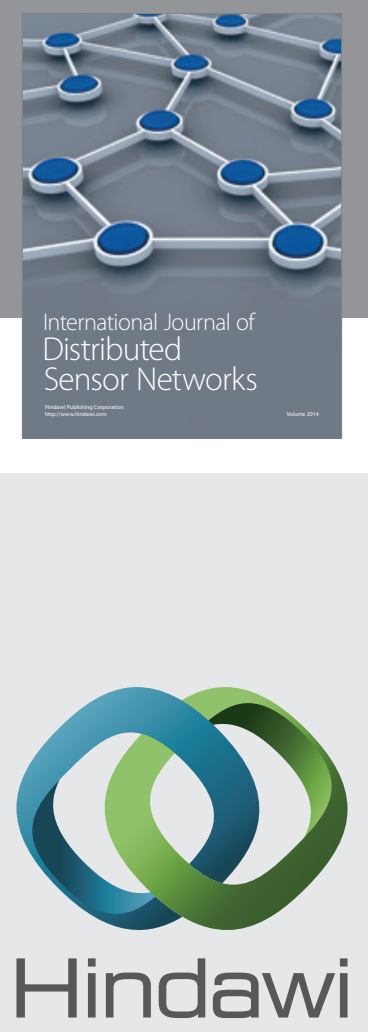

Submit your manuscripts at

http://www.hindawi.com
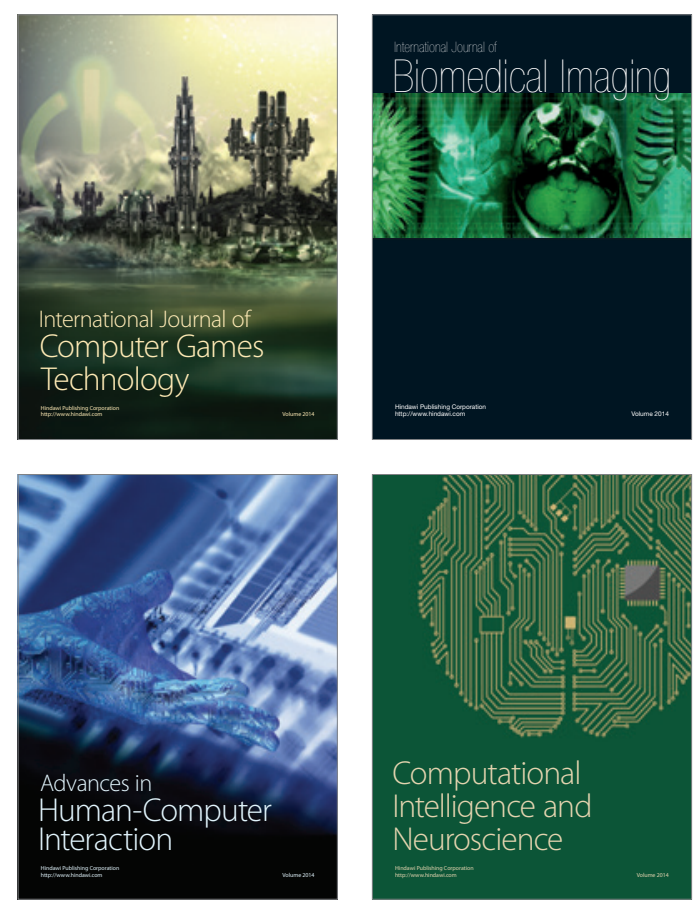
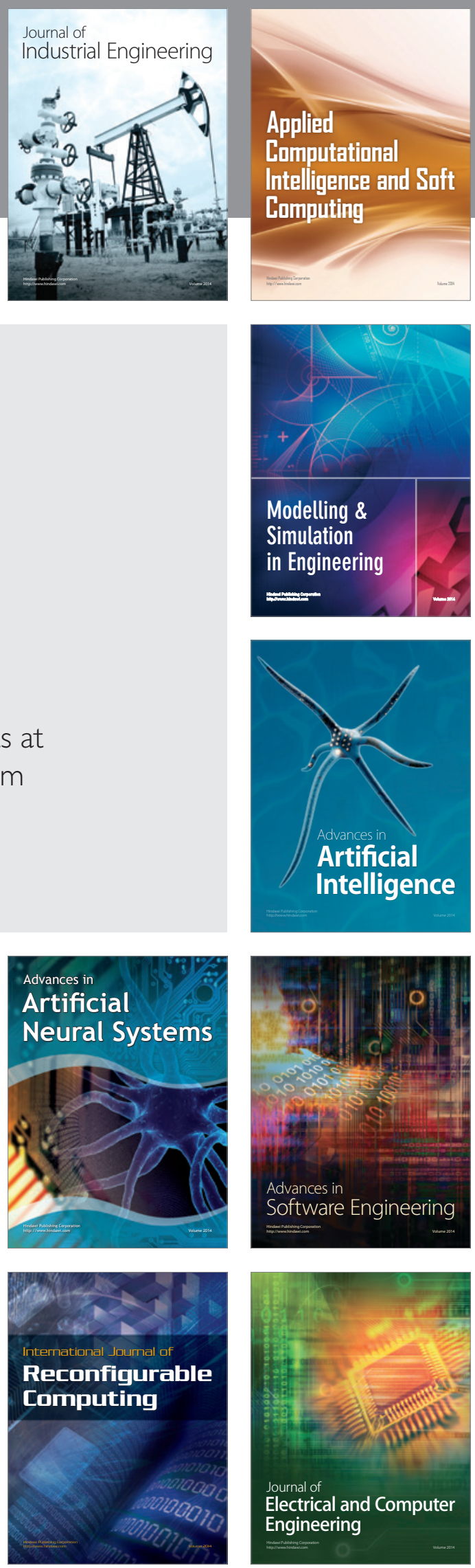